Invited paper

\title{
Wavelength-Tunable, Passively Mode-Locked Erbium-Doped Fiber Master-Oscillator Incorporating a Semiconductor Saturable Absorber Mirror
}

\author{
Luis A. Vazquez-Zuniga and Yoonchan Jeong* \\ Laser Engineering and Applications Laboratory, Department of Electrical and Computer Engineering, \\ Seoul National University, Seoul 151-744, Korea
}

(Received February 7, 2013 : revised March 16, 2013 : accepted March 18, 2013)

\begin{abstract}
We briefly review the recent progress in passively mode-locked fiber lasers (PMLFLs) based on semiconductor saturable absorber mirrors (SESAMs) and discuss the detailed characterization of a SESAM-based, passively mode-locked erbium-doped fiber (EDF) laser operating in the $1.5-\mu \mathrm{m}$ spectral range for various configurations. A simple and compact design of the laser cavity enables the PMLFL to generate either femtosecond or wavelength-tunable picosecond pulses with high stability as the intra-cavity filtering method is altered. All the cavities investigated in our experiments present self-starting, continuous-wave mode-locking with no Q-switching instabilities. The excellent stability of the source eventually enables the wavelength-tunable PMLFL to be used as a master oscillator for a power-amplifier source based on a large-core EDF, generating picosecond pulses of $>10-\mathrm{kW}$ peak power and $>100-\mathrm{nJ}$ pulse energy.
\end{abstract}

Keywords : Mode-locked lasers, Erbium fiber lasers, Semiconductor saturable absorber mirrors, Picosecond
pulses

OCIS codes : (140.3510) Lasers, fiber; (140.3500) Lasers, erbium; (140.4050) Mode-locked lasers; (320.5550) Pulses

\section{INTRODUCTION}

Fiber-based, passively mode-locked (ML) laser sources have become important tools for a variety of scientific and engineering applications [1-7] because of their simplicity and compactness in obtaining a broad range of operation in terms of pulsewidth (fs to ps) as well as pulse repetition rate (kHz to $\mathrm{GHz}$ ). Since they are primarily based on fiber laser technology, they also allow for excellent power scalability, large spectral bandwidth, and good spatial mode quality [8]. In fact, passive mode-locking (ML) is an all-optical technique that relies on the intensity-dependent cavity loss mechanism via the interaction of intensity fluctuations and nonlinearities of the laser cavity medium without having an external control for the signal [9]. Over the last decades, various passive ML mechanisms or devices, which include nonlinear polarization evolution [10-12], nonlinear amplifier loop mirrors [13, 14], semiconductor saturable absorber mirrors (SESAMs) [15-37], more recently carbon nanotubes
(CNTs) [38, 39], graphene [40-42], etc have been introduced to fiber lasers. The first two methods rely on the "virtual" saturable absorbers via the Kerr nonlinearity of fiber combined with a polarizer. While these types of saturable absorbers present fast recovery times which allow for the formation of sub-hundred fs pulses [9], such methods in general do not readily offer self-starting ML and long-term stability to the system. On the other hand, the inclusion of "real" saturable absorbers, such as the last three devices, can radically enhance the cavity loss modulation in the cavity, thereby leading to self-starting ML and also providing high stability to the system [16].

Among the three real saturable absorbers, CNT- and graphene-based saturable absorbers have received a lot of attention over the recent years [38-42] since they can be relatively easily fabricated and integrated with optical fibers [39]. Furthermore, CNT-based saturable absorbers can allow for the formation of very short pulses because of their fast recovery times [39], and on the other hand graphene-based

\footnotetext{
*Corresponding author: yoonchan@snu.ac.kr

Color versions of one or more of the figures in this paper are available online.
} 
saturable absorbers offer a very broad spectral range of operation [39]. While they have great potential to be used for passively ML fiber lasers (PMLFLs), their development is still in the early stages [40-42]. In contrast, SESAMs have already been proven as commercial products, offering standardized performance as a saturable absorber with excellent long-term reliability [16]. In this light, SESAMs are absolutely suitable for use in master-oscillator lasers, which invariably require superior long-term reliability in the first place.

Here, we present a brief review of the recent development of PMLFLs based on SESAMs, their fundamental characteristics, and the detailed characterization of a SESAM-based wavelengthtunable PMLFL that can generate optical pulses of fs to ps, building on our previous investigations [36, 37]. In particular, we analyze the formation of the pulses with respect to the filter bandwidth as well as the tunability across the $1.5-\mu \mathrm{m}$ spectral range. For this, a sigma-cavity configuration is investigated for two different types of tunable filters. The excellent stability of the developed PMLFL eventually enables it to be used as a master oscillator for a poweramplifier source based on a large-core erbium-doped fiber (EDF), generating picosecond pulses of $>10-\mathrm{kW}$ peak-power and $>100$-nJ pulse-energy, which should be very useful for second-harmonic generation or supercontinuum generation, for example.

\section{REVIEW OF SESAM AND SESAM-BASED PMLFLS}

Since the first development of a SESAM in 1992 [15], it has become an essential intra-cavity component for passive ML lasers [14, 15]. One of the reasons for their successful, commercial growth relies on the fact that their optical properties can be engineered to be fitted for a broad range of laser cavity designs [15-37]. While SESAMs were initially utilized to resolve the Q-switching instability problems in ML solid-state lasers suffering from excessively long upperstate lifetimes [15, 43], their uses in PMLFLs have also offered an excellent route forward to realizing stable, selfstarting ML mechanisms [18]. Hence, the progress in SESAM technology has paved the way for building compact and stable ultrashort-pulsed lasers in PMLFLs as well as in bulk-type solid-state lasers [15-37].

In general, semiconductor materials are good saturable absorbers because of the interplay of carriers between the valence and conduction bands: For instance, when there is incoming light to a semiconductor material, it will excite carriers (electrons or holes) from the valence band to the conduction band [16] if it has sufficient photon energy exceeding the bandgap energy of the semiconductor material, which will, in fact, result in the absorption of the incoming light. The absorption rate may remain unchanged if the intensity of the light (photon intensity) is sufficiently low. However, if the photon intensity is high, the absorption will be saturated since the carriers in the valence band are temporarily depleted until the excited carriers are released back into the valence band from the conduction band, thereby leading to a temporal reduction of the net loss of the device to the incoming light. Since these devices are usually integrated with mirror structures, in such cases the reflectivity of the device will increase within the relaxation time of the semiconductor material as its optical loss reduces at high photon intensities. These types of devices based on semiconductor materials are known as SESAMs [16, 17].

A typical structure of a SESAM is shown in Fig. 1. A bottom Bragg reflector is formed by a group of N-pair quarterwaveplates of GaAs/AlAs grown on a semiconductor substrate GaAs, for example [15]. The Bragg wavelength of the reflector is primarily determined by the operating wavelength of the target laser. This mirror structure is then followed by a semiconductor saturable absorber and a secondary top reflector. The secondary top reflector can be formed either by the simple saturable absorber/air interface or by a Bragg reflector. It should be noted that the optical pulse reflected by the SESAM will undergo a significant decrease in its leading edge because the saturable absorber initially keeps giving rise to high loss until the loss is saturated by the central part of the optical pulse of high intensity. Consequently, optical pulses will become steeper and steeper while being repeatedly reflected by the SESAM, which, in fact, functions as a high-intensity-pass shutter in a laser cavity.

In general, the output spectrum of a fiber laser can be formed of many longitudinal modes, i.e., many spectral components spaced by the fundamental repetition rate that is the inverse of the cavity round-trip time. In a homogeneously-broadened gain medium like an EDF, such modes compete for gain resources within the finite gain bandwidth given in the cavity, and only the mode which sees the highest net gain will dominate over the other modes. For the case in which the longitudinal modes are independent and random in phase, the instantaneous amplitude of the laser output will also oscillate randomly in the time domain, thereby only being able to emit steady average output power. However, when the phases of all the longitudinal modes

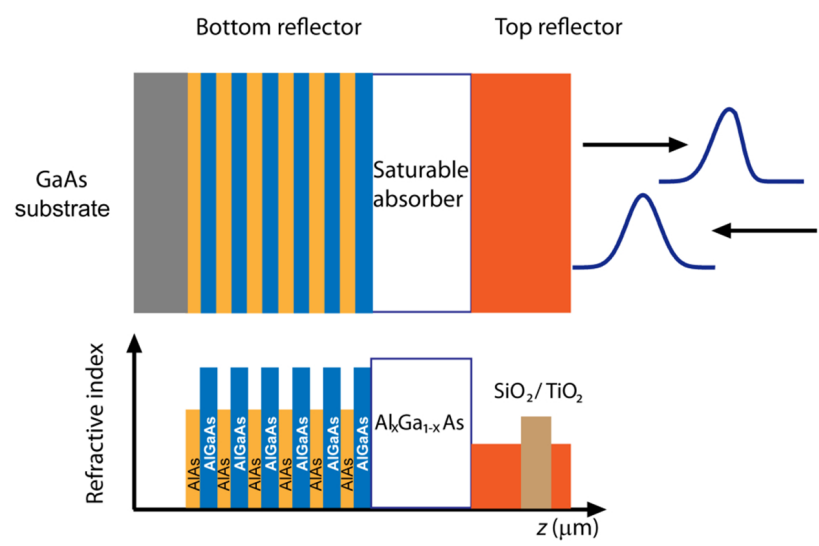

FIG. 1. Schematic of a semiconductor saturable absorber mirror [15]. 
are fixed to a constant value, the laser output will emit a pulse train oscillating at the fundamental repetition rate or its higher-order harmonics [9]. In order to have such a regularly oscillating pulse train, i.e. to lock the longitudinal modes, there must be a mechanism to initiate the intensity fluctuation and clean up the undesirable emissions between the pulse intervals. This can be achieved by an active method, e.g. by an electro-optic modulator or by a passive method, e.g., by a saturable absorber like a SESAM. As described earlier, the fast saturation and recovery of the loss mechanism obtained from a SESAM invariably favors regularly oscillating pulses formed of phase-locked longitudinal modes of the cavity. That is, the use of a SESAM in a homogeneouslybroadened laser cavity can readily lead to passive ML.

As described above, the properties of SESAMs can be tuned and engineered for many particular purposes of target lasers, and thus, there are a variety of choices for them. Due to the dramatic growth in semiconductor technology, SESAMs of various characteristics are mostly commercially available [44]. Thus, it conversely becomes the first and foremost task to choose a correct SESAM design in order to achieve a good laser performance or to avoid Q-switching instabilities. (The latter is a common issue for lasers having gain media of excessively long upper-state lifetimes [17].) For example, if modulation instabilities are not present in the laser cavity, such as in stretched-pulse laser cavities [20], a SESAM with high modulation depth $(\Delta R)$ is desirable to enhance the mechanism of pulse narrowing and to provide sufficiently large perturbation for self-starting ML. Yet, a high modulation depth leads to an increment of the non-saturable loss $\left(A_{n s}\right)$, which can eventually give rise to Q-switching instabilities [46]. Therefore, a SESAM with low modulation depth is sometimes desirable to suppress the Q-switching instabilities, thereby enhancing the stability of continuous-wave (CW) ML. (It should be noted that we use the term CW ML to define the operation of a ML laser whose pulse energy or average power is constant. This means that there are no large amplitude fluctuations in the output signal as can be seen in Q-switched ML regimes, for example.) Therefore, there must be a certain trade-off in determining the modulation depth of a SESAM, so that the stability criterion has been investigated for passively ML lasers $[45,46]$ : If the relaxation time of the saturable absorber is much shorter than the cavity round-trip time, the minimum intra-cavity pulse energy for enabling stable CW ML for a linear cavity is given by [45]:

$$
\begin{aligned}
& E_{P, c}=\left(F_{s a t, L} A_{e f f, L} F_{\text {sat }, A} A_{e f f, A} \Delta R\right)^{1 / 2} \\
& F_{\text {sat. } L}=h v /\left[2\left(\sigma_{e}+\sigma_{a}\right)\right]
\end{aligned}
$$

where $F_{\text {sat }, L}$ is the gain saturation fluence, $h_{v}$ is the lasing photon energy, $\sigma_{e}$ and $\sigma_{a}$ are the emission and absorption cross-sections of the gain medium at the lasing wavelength, $F_{\text {sat }, A}$ is the SESAM saturation fluence, $A_{\text {eff }, L}$ and $A_{\text {eff }, A}$ are the effective laser mode areas in the gain medium and on the SESAM, respectively. According to Eq. (1), the critical intra-cavity pulse energy mainly depends on five different parameters, which must be carefully determined to maximize the overall stability. In particular, $A_{\text {eff } f,}$, i.e., the effective laser mode area on the SESAM can readily be modified if one adjusts the beam size incident on the SESAM [45], which can be a flexible way to improve the stability of CW ML.

Since the first GaAs-based SESAM devices were developed for $800-\mathrm{nm}$ radiation [16], various semiconductor materials and compositions have been developed for other infrared ranges [18-37]: For 0.9-1- $\mu \mathrm{m}$ radiation, SESAMs are mostly fabricated in a form of an InGaAs multiple-quantumwell structure grown on top of a GaAs/AlAs Bragg mirror $[23,30]$. With such devices ML lasers based on neodymium $(\mathrm{Nd})$ - and ytterbium $(\mathrm{Yb})$-doped fibers have been demonstrated $[23,30]$. Other types SESAMs based on GaInNAs structures for the $1-\mu \mathrm{m}$ and $1.3-\mu \mathrm{m}$ wavelength regions have also shown good performance for ML-lasers [22, 26, 32]. These SESAMs utilize a GaInNAs-based structure grown on top of a GaAs/AlAs Bragg mirror. With such a device, a ML laser based on a bismuth (Bi)-doped fiber has been demonstrated [32]. For radiations around $1.5 \mu \mathrm{m}$ or above, InP-based [19, 25, 33] SESAMs and GaAs-based diluted nitride [27] SESAMs are commonly used: An InGaAs/InP Bragg mirror with an absorber made of InGaAs quantum wells has been used to generate 190-fs pulses from a thulium (Tm)-doped fiber laser operating at $\sim 2 \mu \mathrm{m}$ [33]. More recently, a ML Tm/Holmium (Ho)-doped fiber laser has been built with help of a SESAM where the absorber material was formed of a group of GaInSb quantum wells grown on top of an AlAsSb-GaSb Bragg mirror [24].

Leveraged by such rapid progress in the SESAM technology, there have been dramatic advances in SESAM-based PMLFLs operating at $\sim 1.5 \mu \mathrm{m}$. This spectral range (which will be discussed throughout this paper) is of great interest because of its inherent "eye-safe" nature together with good transmission in both silica and air. Consequently, such PMLFLs have been investigated for many novel scientific and engineering applications, such as free-space communication, range finding, LIDAR, and medicine [1-6], besides the optical fiber telecommunications. Thus, a variety of compact and self-starting PMLFLs in femtosecond and picosecond regimes have been demonstrated: For example, in the femtosecond regime, 320-fs soliton-like pulses with 40-pJ pulse energy have been generated in a simple linear cavity with an InGaAsP saturable absorber [19], while sub-500-fs pulses with repetition rates as high as $300 \mathrm{MHz}$ have been generated with an $\mathrm{InGaAs} / \mathrm{InP}$ saturable absorber [34], which could also operate at higher harmonics by increasing the repetition rate up to $2 \mathrm{GHz}$. In the picosecond regime, saturable absorbers based on GaInNAs/GaAs have been used to generate 7.6-ps noise-like pulses from an Er/Yb-doped fiber [35] and 1.2-ps soliton-like pulses from an EDF [27].

In addition, saturable absorbers have also been investigated 
for various other purposes: For example, an inclusion of linear loss elements in cavity combined with an InGaAsP-based saturable absorber could avoid multiple-pulse formation at high pump powers [18]. Hybrid configurations incorporating a pair of fast and slow saturable absorbers have been proposed to provide a reliable self-starting ML mechanism [21], where pulses with 250-pJ pulse energy were generated without having multiple-pulse breakup at high pump powers. SESAMs have also been used for pulse shaping in fiber lasers operating at fundamental repetition rates above $2 \mathrm{GHz}$ [28].

Hence, it has become clear that SESAMs have vitalized PMLFLs with novel characteristics as well as offering robust reliability, thereby having now been proven as a mature technology [16]. Here we discuss in the following sections the detailed configurations and characteristics of a SESAM-based, wavelength-tunable PMLFL.

\section{CAVITY CONFIGURATIONS AND SESAM CHARACTERISTICS}

A sigma configuration proposed for our PMLFL is shown in Fig. 2. The laser consists of $2 \mathrm{~m}$ of an EDF (Fibercore), which has a small-signal absorption rate of $\sim 37 \mathrm{~dB} / \mathrm{m}$ at $1530 \mathrm{~nm}$ and is pumped by a single-mode laser diode (LD) of a total power of $140 \mathrm{~mW}$ at $1480 \mathrm{~nm}$ through a 1480/1550 wavelength-division-multiplexed (WDM) coupler. A second 1480/1550 WDM coupler is spliced to the other end of the EDF in order to remove the unabsorbed pump light from the cavity. Two sets of polarization controllers, PC1 and PC2, are inserted before and after a fiberized polarization beam splitter (PBS) to introduce an appropriate phase bias for locking the longitudinal modes of the ring cavity as well as to adjust the output coupling ratio through the PBS. PC1 is comprised of three waveplates (a quarterwaveplate, a half-waveplate, and a quarter-waveplate), while PC2 is of only two waveplates (a quarter-wave-plate and a half-waveplate). A 95:5 tap coupler is spliced to PC2 to monitor the intra-cavity signal, which is followed by a 3-port circulator. The fiber end of port-2 of the circulator is angle-cleaved, and its output signal is collimated and launched onto a SESAM (BATOP Optoelectronics, see Table 1 for

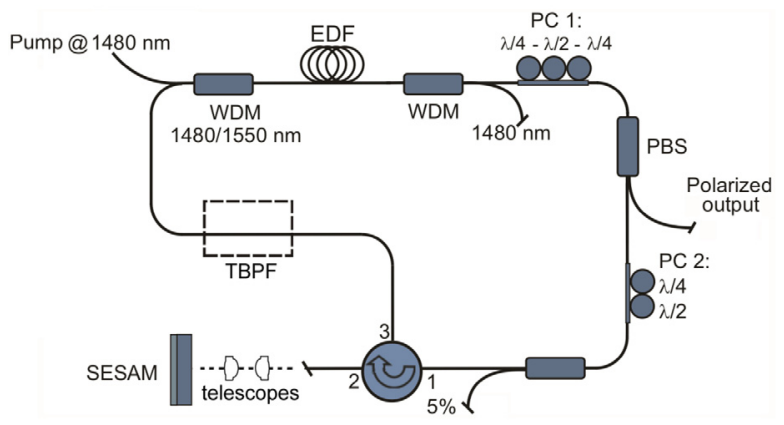

FIG. 2. Schematic of the PMLFL with a SESAM. The configuration is tested for different TBPFs.
TABLE 1. SESAM specifications

\begin{tabular}{l|c}
\hline \hline \multicolumn{1}{c|}{ High reflection band } & $1550-1560 \mathrm{~nm}$ \\
\hline Absorbance & $23 \%$ \\
\hline Modulation depth $(\mathrm{D} R)$ & $14 \%$ \\
\hline Non-saturable loss $\left(A_{n s}\right)$ & $9 \%$ \\
\hline Saturation fluence $\left(F_{\text {sat, } A}\right)$ & $25 \mathrm{~mJ} / \mathrm{cm}^{2}$ \\
\hline Relaxation time constant $\left(t_{R}\right)$ & $\sim 2 \mathrm{ps}$ \\
\hline Damage threshold & $500 \mathrm{MW} / \mathrm{cm}^{2}$ \\
\hline
\end{tabular}

TABLE 2. Specifications of the two TBPFs used in the PMLFL

\begin{tabular}{l|c|c}
\hline \hline \multicolumn{1}{c|}{ Parameter } & $\begin{array}{c}\text { TBPF1 } \\
\text { (Alnair Labs) }\end{array}$ & $\begin{array}{c}\text { TBPF2 } \\
\text { (SANTEC) }\end{array}$ \\
\hline $\begin{array}{l}\text { Wavelength tuning range } \\
\text { [nm] }\end{array}$ & $1530-1610$ & $1535-1565$ \\
\hline Bandwidth @ -3dB [nm] & $0.1-13.0$ & 1.3 \\
\hline Bandwidth @ -20 dB [nm] & $0.3-13.25$ & $<15$ \\
\hline Insertion loss [dB] & $3.5-7.0$ & 1.5 \\
\hline
\end{tabular}

details) through a pair of aspheric lenses, which facilitates adjusting the incident pulse fluence onto the SESAM via varying its illuminated area. Finally, to close the sigma cavity, the output port of the circulator is spliced to the input port of the first 1480/1550 WDM through a reconfigurable section which may or may not include a fiberized tunable band-pass filter (TBPF). For the fiberized TBPF we investigate two different types: One is a novel type of tunable filter that can also offer a bandwidth-adjustable function (TBPF1: Alnair Labs, see Table 2 for details). The other is a low-loss fiberized optical tunable filter (TBPF2: SANTEC, see Table 2 for details) with a fixed filter bandwidth $\left(\Delta \lambda_{F}\right)$ of $1.3 \mathrm{~nm}$ in full width at half maximum (FWHM).

Thus, the total cavity length can be $10.92 \mathrm{~m}$ (without a TBPF), $15.54 \mathrm{~m}$ (with TBPF1), or $15.06 \mathrm{~m}$ (with TBPF2), unless stated otherwise. We estimate the corresponding average group velocity dispersion (GVD) parameter of the resultant cavity to be $\beta_{2}=-0.1894 \mathrm{ps}^{2}, \beta_{2}=-0.2464 \mathrm{ps}^{2}$, or $\beta_{2}=-0.2354 \mathrm{ps}^{2}$, respectively. The pulses generated by the laser cavity are primarily characterized with an optical spectrum analyzer (OSA), a radio-frequency (RF) spectrum analyzer, an autocorrelator based on background-free (noncollinear) second-harmonic generation, and a frequency-resolved optical gating (FROG) system (Southern Photonics HR150). It is noteworthy that the FROG system has temporal and optical resolutions of $26.66 \mathrm{fs}$ and $0.05 \mathrm{~nm}$, respectively.

\section{EXPERIMENTAL RESULTS}

\subsection{Femtosecond-pulsed Regime}

We first characterized the laser system without incorporating 
a TBPF. That is, the output port of the circulator was directly spliced to the input port of the first 1480/1550 WDM though a standard single-mode fiber (SMF28, Corning). In this configuration, the laser presented stable, self-starting ML pulses centered at $1556 \mathrm{~nm}$ circulating at the fundamental cavity frequency of $18.30 \mathrm{MHz}$ for the pump power $\left(P_{\text {pump }}\right)$ of $\sim 37 \mathrm{~mW}$, which remained stable for pump power of up to $\sim 48 \mathrm{~mW}$ while the pulsewidth slightly varied with the pump power. Above this power level the laser output gave rise to unstable, multiple pulses, which is a typical characteristic of soliton lasers with excessive gain [12]. In particular, for $P_{\text {pump }}=\sim 43 \mathrm{~mW}$ the laser generated the shortest pulses in the femtosecond regime with pulse energy of $105 \mathrm{pJ}$, peak power of $\sim 298 \mathrm{~W}$, and average output power of $\sim 1.9 \mathrm{~mW}$. Fig. 3 shows its characteristics measured in the temporal and spectral domains. The measured pulsewidth was given by $\sim 310$ fs (FWHM), assuming a sech $^{2}$ profile, and the spectral bandwidth was given by $\sim 9.72 \mathrm{~nm}$ (FWHM), thereby yielding the time-bandwidth product (TBP) of $\sim 0.372$. One can see the Kelly sidebands formed by co-propagating dispersive waves in the optical spectrum shown in Fig. 3(b), which clearly indicates a soliton-like nature of the pulses generated in this laser cavity [47]. Based on the calculation method given in Ref. [48] together with the RF spectrum measured at the fundamental cavity frequency $(18.30 \mathrm{MHz})$ shown in Fig. 3(c), we characterized the amplitude fluctuation $(\Delta E / E)$ to be $0.091 \%$, which highlights the high stability of the laser output. We also emphasize that even with the relaxation time constant $\left(\tau_{\mathrm{R}}\right)$ of the saturable absorber of $\sim 2$ ps (see Table 1), the formation of such a short pulse was absolutely possible due to the fact that pulse formation was fundamentally "solitonic" because the captivity was well in the anomalous regime, i.e., $\beta_{2}<0$. In fact, the contribution of the saturable absorber was primarily to stabilize the ML of the cavity modes [46].

According to the soliton area theorem [3], the pulse energy and pulsewidth are related to the average GVD and nonlinearity of the cavity as follows:

$$
\begin{aligned}
& E_{S}=\frac{3.53 \sqrt{\left|D_{2}\right|}}{\gamma L} \\
& \Delta \tau=\sqrt{\left|D_{2}\right|}
\end{aligned}
$$

where $D_{2}$ is defined by $\beta_{2} L, L$ is the cavity fiber length, and $\gamma$ is the average nonlinear coefficient for the cavity fiber. Based on Eqs. (3) and (4), one can readily see that the product of the pulsewidth and the peak power remain constant for a given cavity condition. This implies that one can adjust the pulsewidth of the PMLFL via changing the total dispersion of the cavity. In Fig. 4, the evolutions of the pulsewidth and TBP are characterized as a function of the cavity dispersion for $P_{\text {pump }}=\sim 43 \mathrm{~mW}$. This characterization was done via adjusting the length of the passive

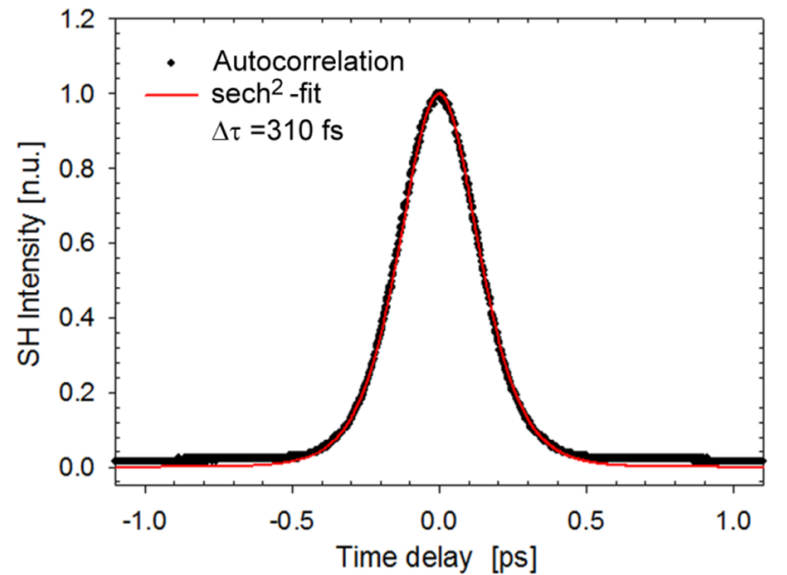

(a)

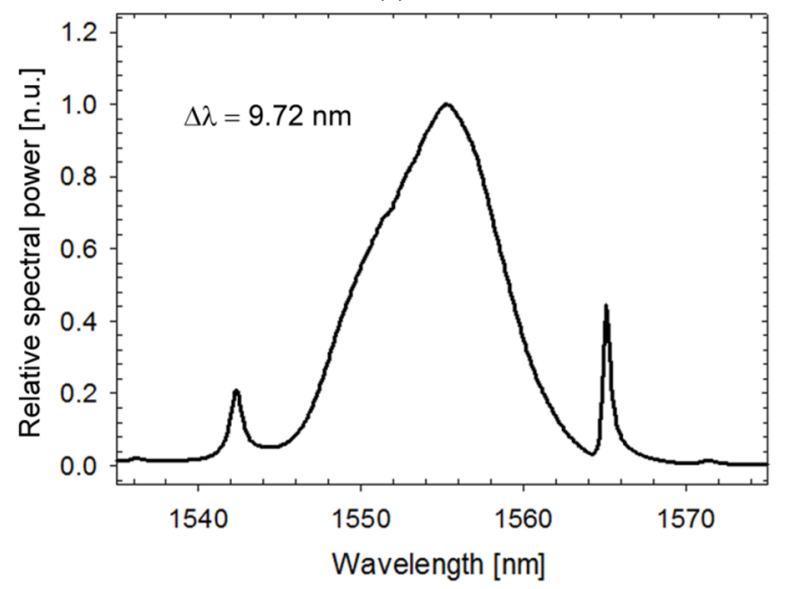

(b)

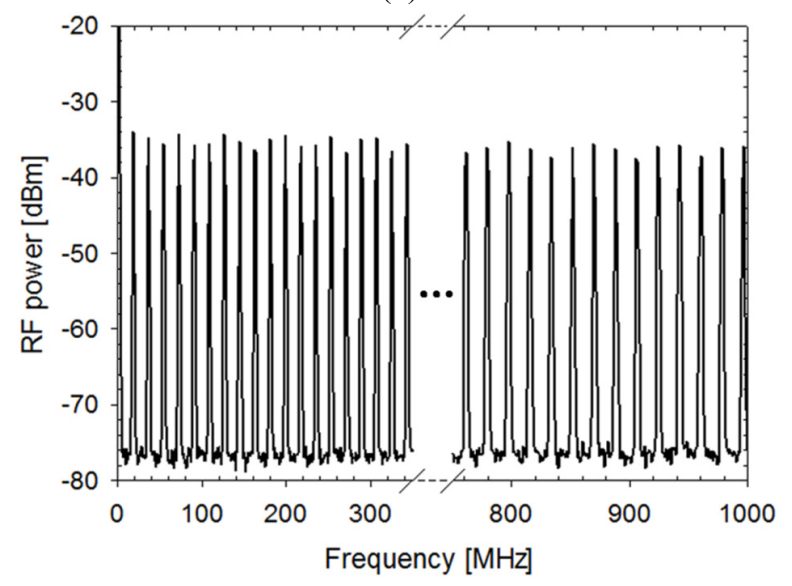

(c)

FIG. 3. (a) Autocorrelation, (b) optical spectrum, and (c) RF spectrum measurements of the output signal for a cavity with $\beta_{2}=-0.1894 \mathrm{ps}^{2}$. n.u.: normalized unit.

fiber section (SMF28) of the cavity. The pulsewidth varied from 248 to $385 \mathrm{fs}$, depending on the average cavity dispersion. In particular, a minimum pulsewidth of $248 \mathrm{fs}$ (FWHM) with a TBP of 0.526 was obtained for $\beta_{2}=-0.078$ $\mathrm{ps}^{2}$. As shown in Fig. 4(a) together with Eq. (3), one can readily see that the pulsewidth became reduced as $\beta_{2} L$ 


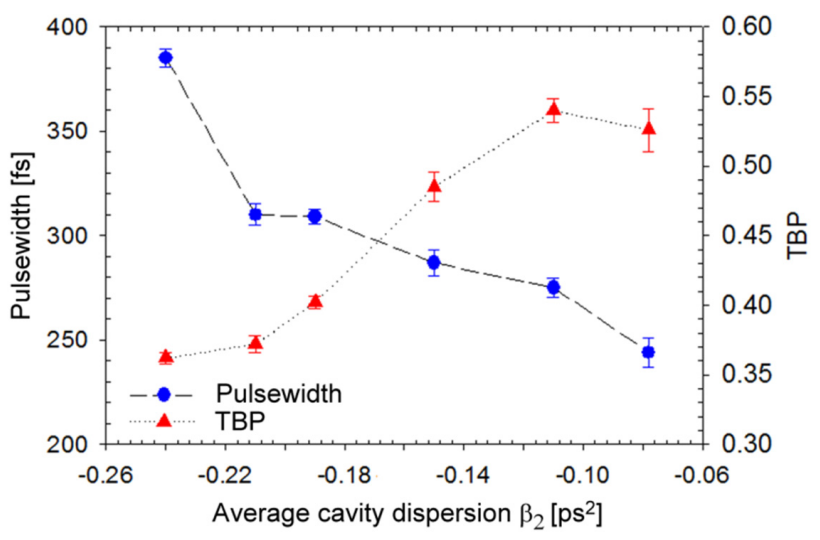

(a)

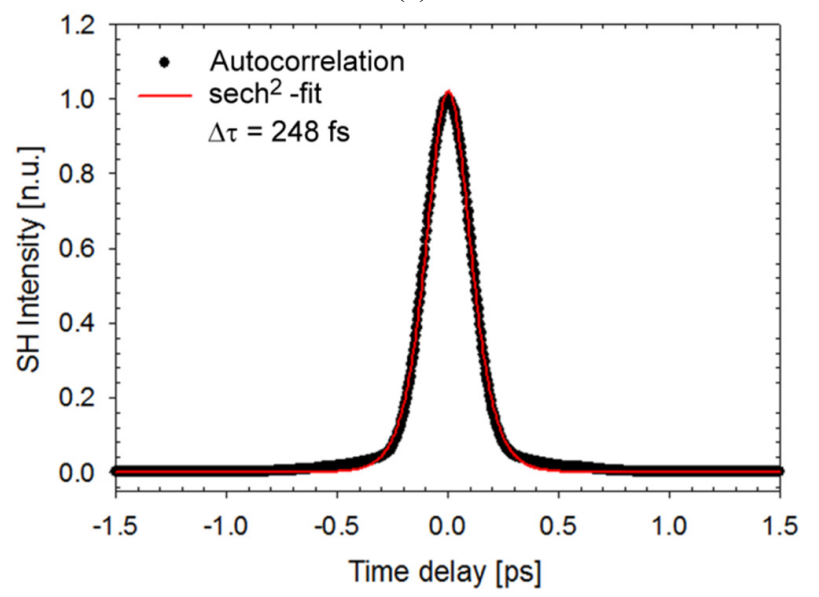

(b)

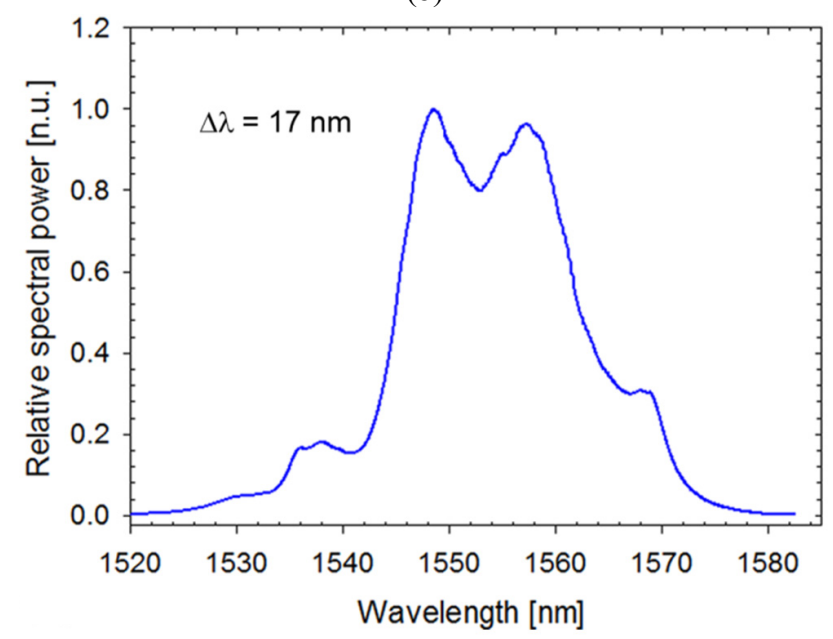

(c)

FIG. 4. (a) Pulsewidth and TBP as a function of $\beta_{2}$, (b) autocorrelation, and (c) optical spectrum of the output signal for $\beta_{2}=-0.078 \mathrm{ps}^{2}$.

decreased. Further reduction of the cavity length was limited by the available length of the passive fiber section in the cavity. In addition, in Fig. 4(c) one can see that when $\beta_{2}$ was adjusted to $-0.078 \mathrm{ps}^{2}$, the Kelly sidebands were nearly suppressed and the shape of the optical spectrum noticeably changed, compared with Fig. 3(b) for $\beta_{2}=-0.1894 \mathrm{ps}^{2}$.
This indicates that the pulse spectrum significantly breathed along the cavity due to the fact that the average GVD was approaching zero [3].

\subsection{Picosecond-pulsed Regime with TBPFs}

We decided to insert a TBPF into the cavity in order to make the PMLFL operate in a wavelength-tunable, picosecond regime, for which we utilized the two TBPFs described in Sec. III. To analyze the influence of the filter bandwidth on the laser characteristics, we firstly investigated the use of TBPF1 because its bandwidth was adjustable while its center wavelength was tuned.

We initially characterized the PMLFL, adjusting the filter bandwidth from $7.2 \mathrm{~nm}$ to $1.5 \mathrm{~nm}$ while keeping the filter center wavelength fixed at $1550 \mathrm{~nm}$. Within this bandwidth range we could obtain stable ML picosecond pulses; however, beyond this range we could see that ML pulses went slightly unstable. For the filter bandwidth of TBPF1 of 7.2 $\mathrm{nm}$, the PMLFL generated stable, self-starting fundamental ML pulses of 2.76-ps pulsewidth, circulating at 12.87 $\mathrm{MHz}$, for which pump power was set to $\sim 30 \mathrm{~mW}$. This pulse regime remained completely locked even when the bandwidth of the filter was narrowed down to $1.5 \mathrm{~nm}$, with which the pulsewidth was broadened to $11.30 \mathrm{ps}$.

Figure 5 shows the experimentally measured FROG traces, the retrieved pulse intensities and phases, and the optical spectra when the filter bandwidth was set to 7.2 $\mathrm{nm}$ and $1.5 \mathrm{~nm}$, respectively. In this regime, the TBP was maintained as high as 0.64 to 0.62 for $\Delta \lambda_{F}=7.2 \mathrm{~nm}$ and $\Delta \lambda_{F}=1.5 \mathrm{~nm}$, respectively. In addition, it is noteworthy that we could see a sinc-like pulse shape having very small satellite side-lobes to the main central pulse in the retrieved pulse intensity profile shown in Fig. 5(a) and 5(b), which we attribute to the fact that the transmission spectrum of the TBPF1 was top-hat-shaped [see Fig. 5(e) and 5(f)]. The retrieval of the phase of the pulse indicates that the pulse was linearly up-chirped, i.e., the leading edge of the pulse was red-shifted while the trailing edge was blue-shifted.

The measured average output powers together with the calculated pulse energies, and measured pulsewidth together with the spectral bandwidth are shown in Fig. 6(a) and Fig. 6(b), respectively, as a function of the bandwidth of TBPF1. The corresponding optical spectra of these measurements are shown in Fig. 6(c), for which the center wavelength of TBPF1 was fixed at $1550 \mathrm{~nm}$ as in Fig. 5. It is also worth mentioning that the PMLFL could also generate pulses of a pulsewidth as short as $\sim 1.3$ ps when the bandwidth of TBPF1 was set to the maximum filter bandwidth of $\sim 13 \mathrm{~nm}$. However, in this case, continuous wavelength tuning beyond $1550 \mathrm{~nm}$ was hardly achieved, which was possibly attributed to the fact that the net gain spectrum of the laser cavity, including the gain of EDF and the loss of TBPF1, was not flat and wide enough to allow for broadband tuning in such a condition.

As a result, we found that the broadest filter bandwidth allowing for continuous wavelength tuning from $1533 \mathrm{~nm}$ 
Wavelength-Tunable, Passively Mode-Locked Erbium-Doped Fiber … Luis A. Vazquez-Zuniga and Yoonchan Jeong 123

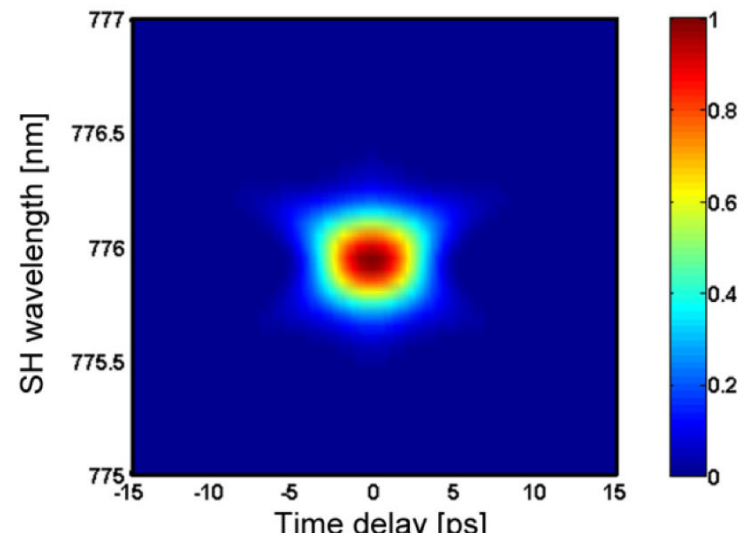

(a)

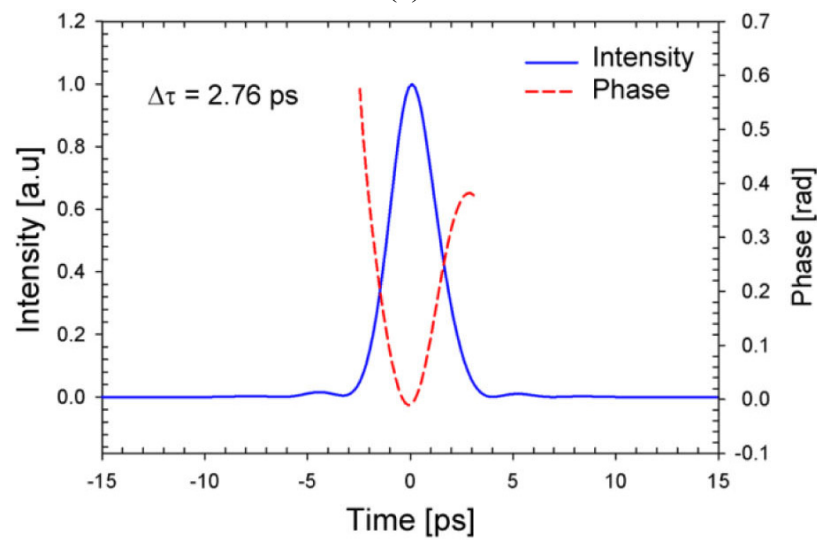

(c)

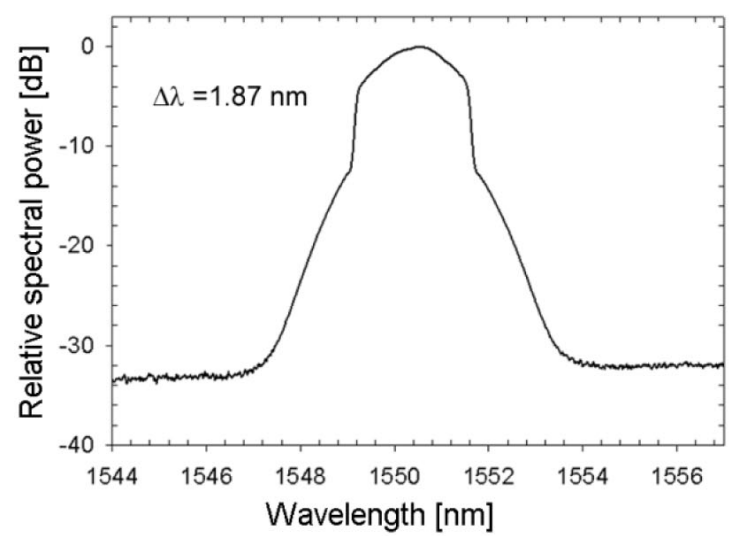

(e)

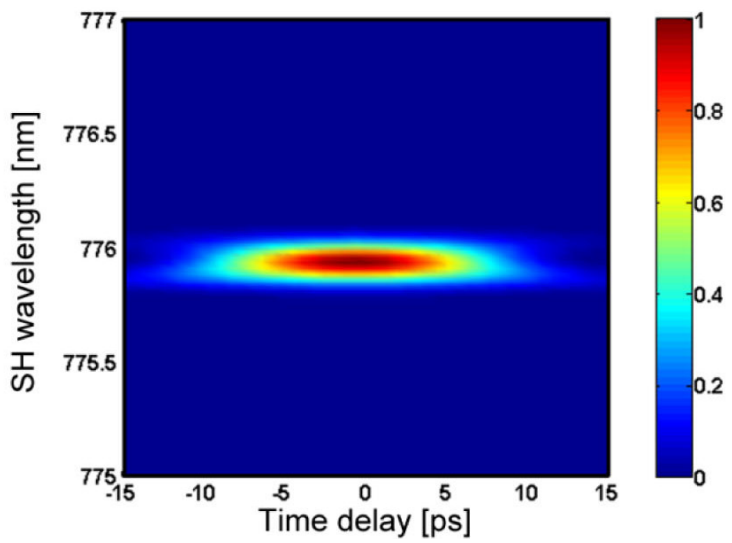

(b)

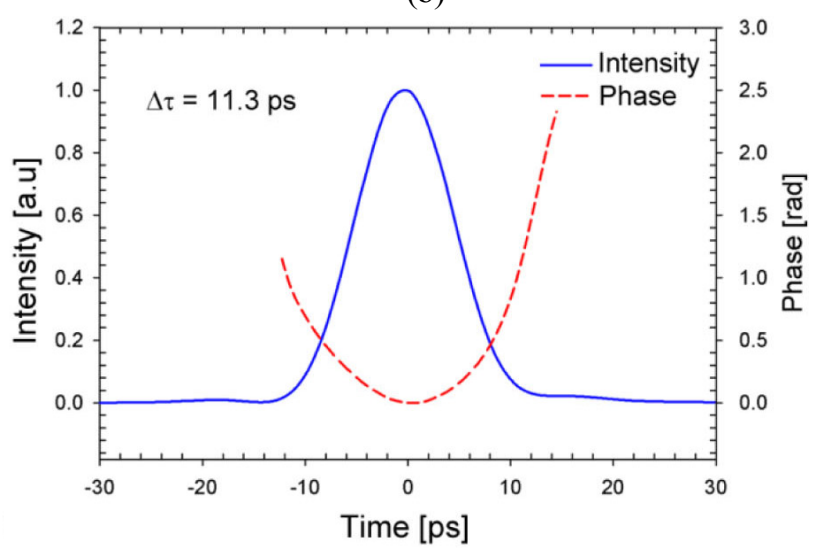

(d)

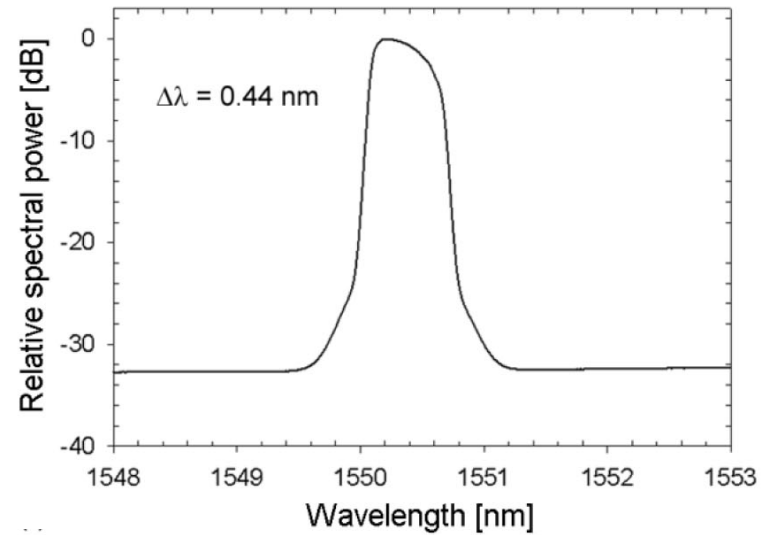

(f)

FIG. 5. (a) and (b): Measured FROG traces. (c) and (d): Retrieved intensity and phase as a function of time. (e) and (f): Measured optical spectra for $\Delta \lambda_{F}=7 \mathrm{~nm}$ (left) and $\Delta \lambda_{F}=1.5 \mathrm{~nm}$ (right). $\mathrm{SH}$ : second harmonic.

to $1555 \mathrm{~nm}$ was given by $\Delta \lambda_{F}=7.2 \mathrm{~nm}$, in which the pump power was fixed at $\sim 35 \mathrm{~mW}$. Fig. 7 shows the measured average output power, pulse energies, and their corresponding optical spectra. For the given tuning range, the PMLFL could generate the average output power of $\sim 0.4 \mathrm{~mW}$ and the pulse energy of $\sim 36 \mathrm{pJ}$. Because of the rather excessive loss $(\sim 5 \mathrm{~dB}$, see Table 2$)$ incurred by th8e inclusion of TBPF1 as well as the reduction of the pulse repetition rate, one can see that the average output power was substantially reduced in comparison with the case without having a TBPF, which was $\sim 1.9 \mathrm{~mW}$ for a similar level of pump power.

Next, we investigated TBPF2 for the wavelength-tuning of the PMLFL in the picosecond regime. The high insertion loss (IL) of $\sim 5 \mathrm{~dB}$ of TBPF1 eventually made it difficult to achieve continuous wavelength-tuning of the PMLFL while keeping the pump power fixed at a constant level. That is, we inconveniently had to adjust the pump power to stabilize 


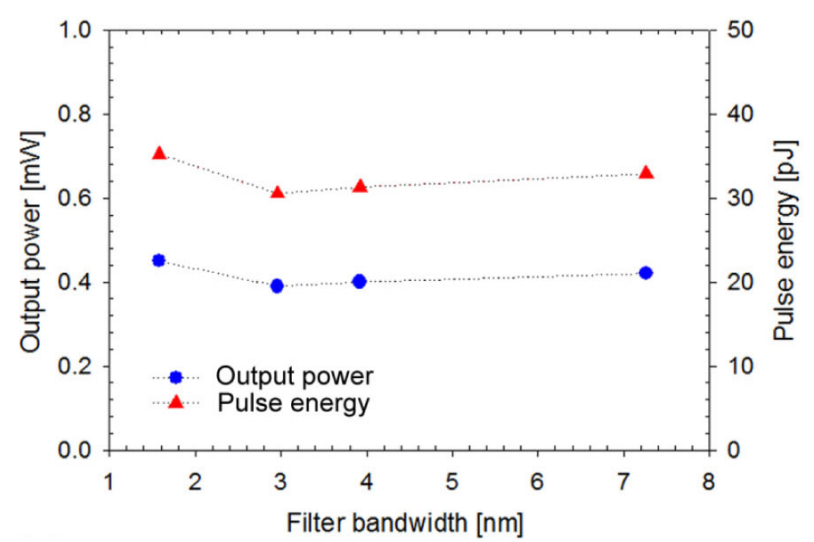

(a)

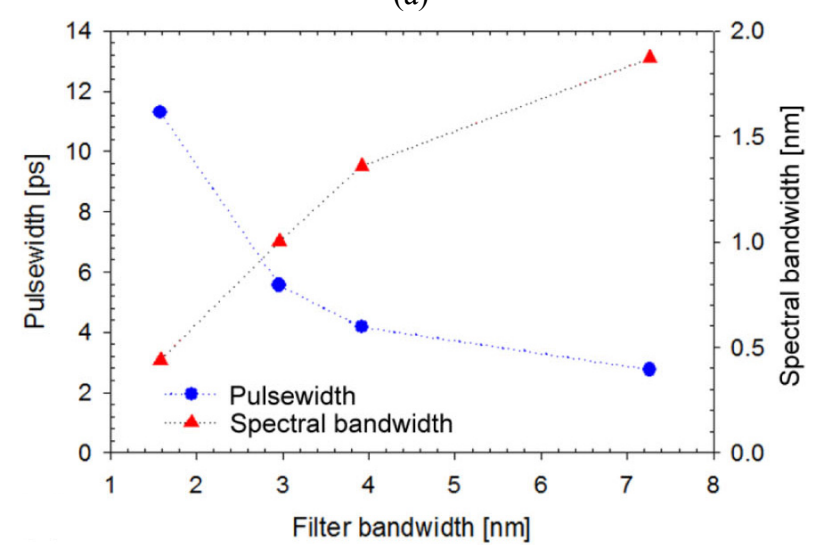

(b)

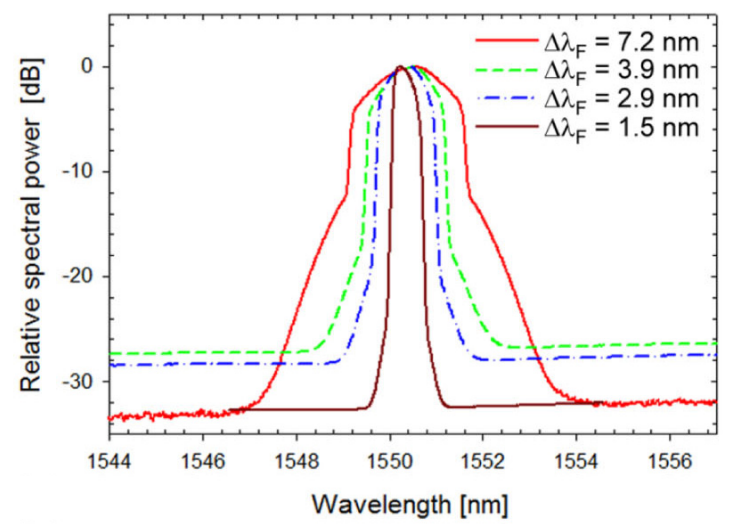

(c)

FIG. 6. (a) Measured average output power and calculated pulse energy. (b) Measured pulsewidth and pulse spectral bandwidth as a function of the filter bandwidth for TBPF1. (c) Corresponding optical spectra of the output signal.

the ML pulses. Thus, we utilized TBPF2 that had a relatively low IL of $\sim 1.5 \mathrm{~dB}$ with a sufficiently large tuning range from $1535 \mathrm{~nm}$ to $1565 \mathrm{~nm}$ although its bandwidth was fixed to $\sim 1.3 \mathrm{~nm}$.

When TBPF2 was initially tuned at $1552 \mathrm{~nm}$, self-starting, fundamental ML pulses were obtained with the pump power as low as $\sim 23 \mathrm{~mW}$ thanks to the low IL of TBPF2 (see Sec. II). Fig. 8 represents the corresponding output

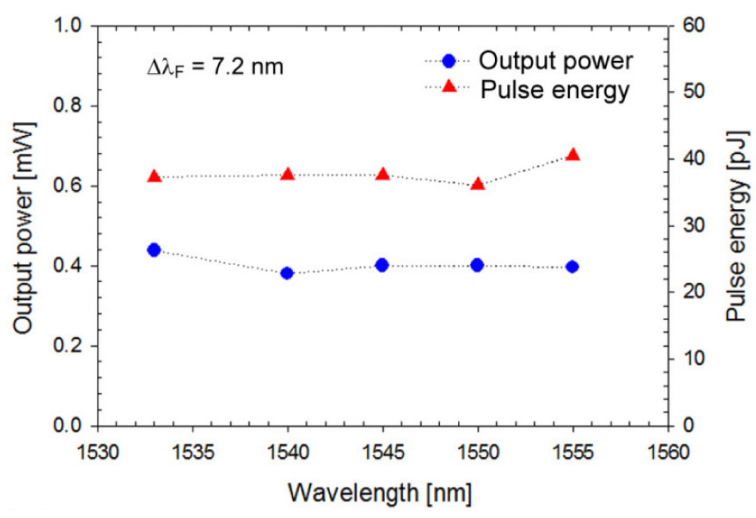

(a)

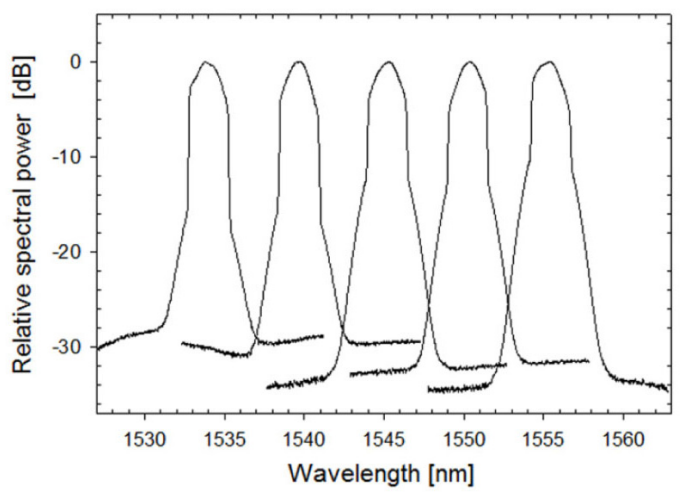

(b)

FIG. 7. (a) Measured average output power and calculated pulse energy over the entire stable tuning range of the PMLFL for $\Delta \lambda_{F}=7.2 \mathrm{~nm}$. (b) Corresponding optical spectra of the output signal.

characteristics of the PMLFL, including the experimentally measured FROG trace of the output pulse, its retrieved intensity and phase, and its spectrum. In the given condition, the pulsewidth, spectral bandwidth, TBP were given by $\sim 8.9$ ps, $\sim 0.48 \mathrm{~nm}$, and 0.53 , respectively. The retrieved phase information of the output pulse indicate that it was also linearly up-chirped similar to the case with TBPF1 (see Fig. 5). In this configuration, we could obtain excellent stability of the ML pulses. Actually, once they were self-started, their ML regime remained completely uninterrupted even with tuning of the TBPF2 throughout the whole tuning range of $1535 \mathrm{~nm}$ to $1560 \mathrm{~nm}$. The pulsewidth of the PMLFL with TBPF2 $\left(\Delta \lambda_{F}=1.3 \mathrm{~nm}\right)$ was given by $\sim 8-10 \mathrm{ps}$, which was in line with the outcomes obtained from the previous characterization of the PMLFL with TBPF1 [see Fig. 6(a)]. In addition, the pulsewidth was slightly adjustable via PC1 and PC2.

Figure 9 shows the pulse characteristics over the entire tuning range of the PMLFL from $1535 \mathrm{~nm}$ to $1560 \mathrm{~nm}$, including its average output power, pulse energy, pulsewidth, spectral bandwidth, and tuned spectra. For this measurement, the pump power was fixed at $\sim 23 \mathrm{~mW}$. We emphasize that thanks to the reduced IL of TBPF2 $(\sim 1.5 \mathrm{~dB})$, the average 


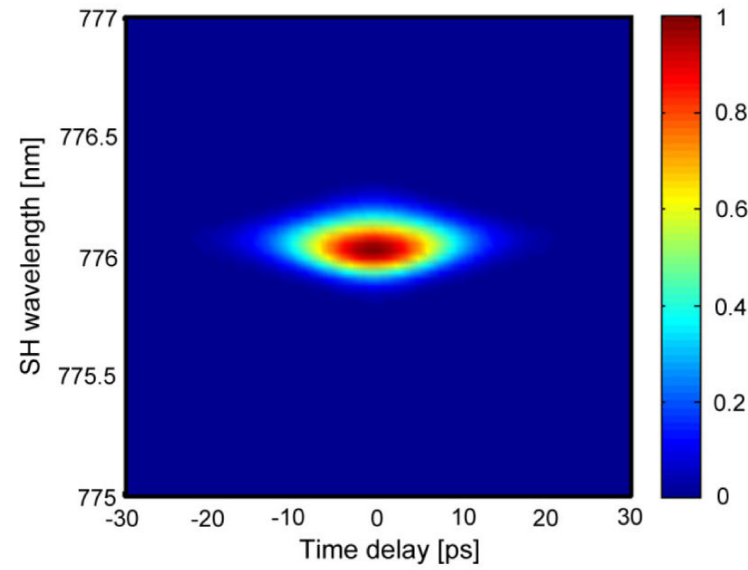

(a)

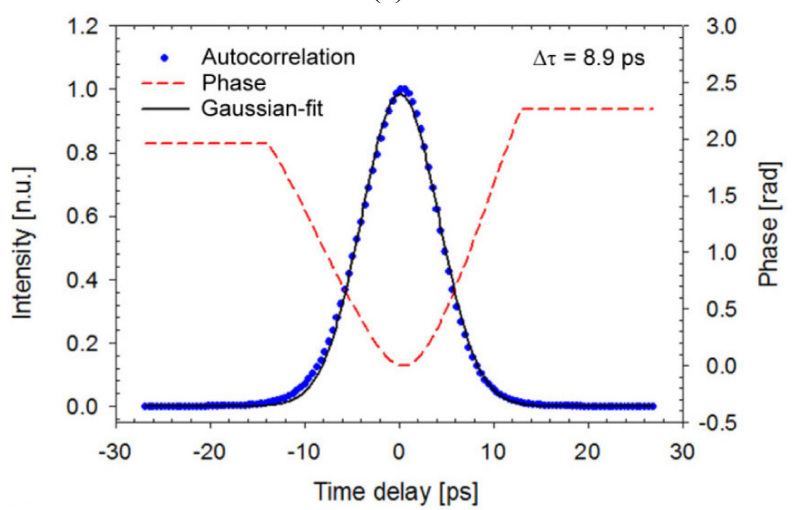

(b)

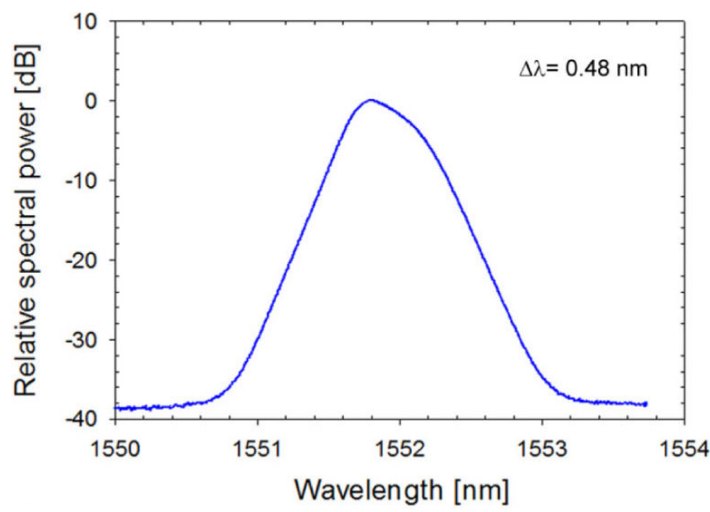

(c)

FIG. 8. (a) Measured FROG traces. (b) Retrieved intensity and phase as a function of time. The retrieved intensity is fitted to a Gaussian pulse with $\Delta \tau=8.9$ ps. (c) Retrieved optical spectrum.

output power was significantly improved to $\sim 1 \mathrm{~mW}$, and this configuration offered much better performance in terms of the pulse stability as well as tuning range, compared to the case with TBPF1.

Here we further characterized this PMLFL, measuring its amplitude noise and timing jitter as follows: Fig. 10 represents its RF spectra measured at the fundamental cavity frequency of $13.28 \mathrm{MHz}$ and at the 10th harmonic

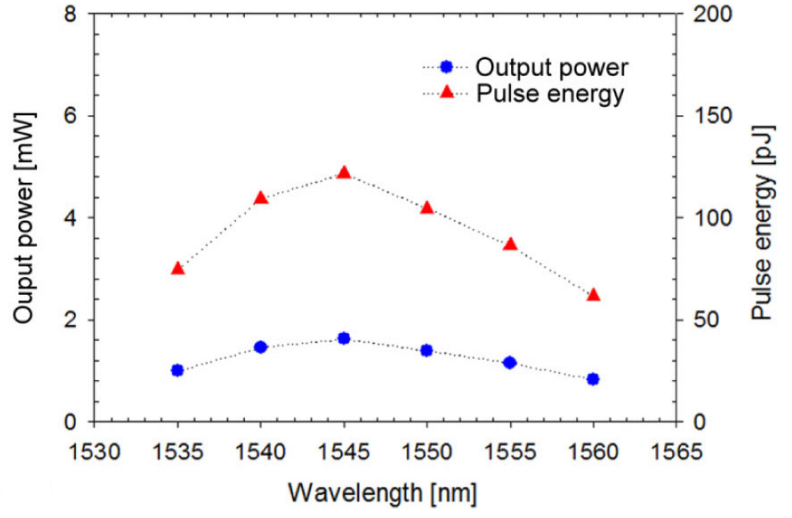

(a)

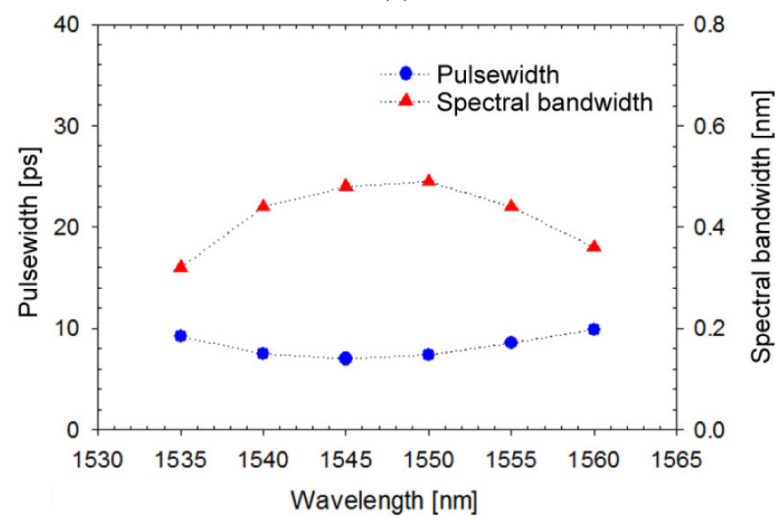

(b)

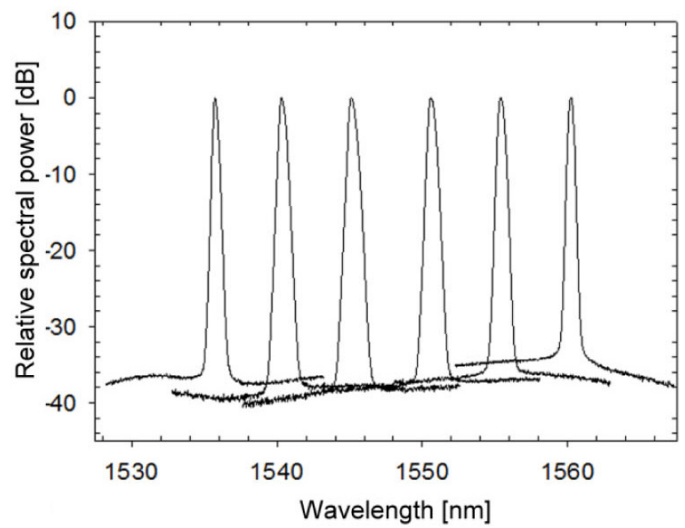

(c)

FIG. 9. (a) Measured average output power and calculated pulse energy. (b) Pulsewidth and pulse spectral bandwidth over the entire tuning range of the PMLFL for $\Delta \lambda_{F}=1.3 \mathrm{~nm}$.

(c) Corresponding optical spectra of the output signal.

frequency of $132.8 \mathrm{MHz}$, respectively, when the PMLFL was tuned at $1552 \mathrm{~nm}$. Based on the RF spectra [48], we characterized the amplitude fluctuation of the PMLFL to be $\Delta E / E=0.47 \%$, which still represents an excellent stability although it slightly increased compared to that in the previous femtosecond regime. The calculated high-frequency timing jitter $(\Delta T)$ of the pulses was given by $6.09 \mathrm{ps}$. Considering the long cavity period (T) of $75.3 \mathrm{~ns}$, this timing jitter represents the time fluctuation $(\Delta T / T)$ of 


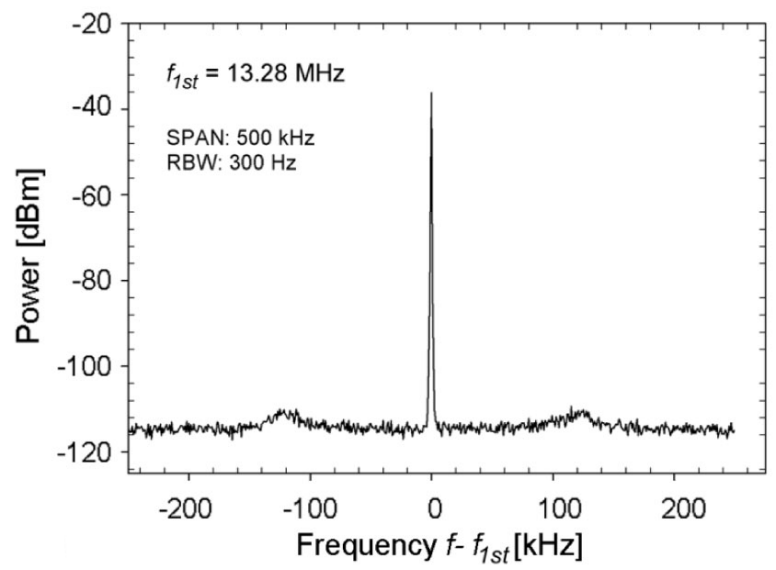

(a)

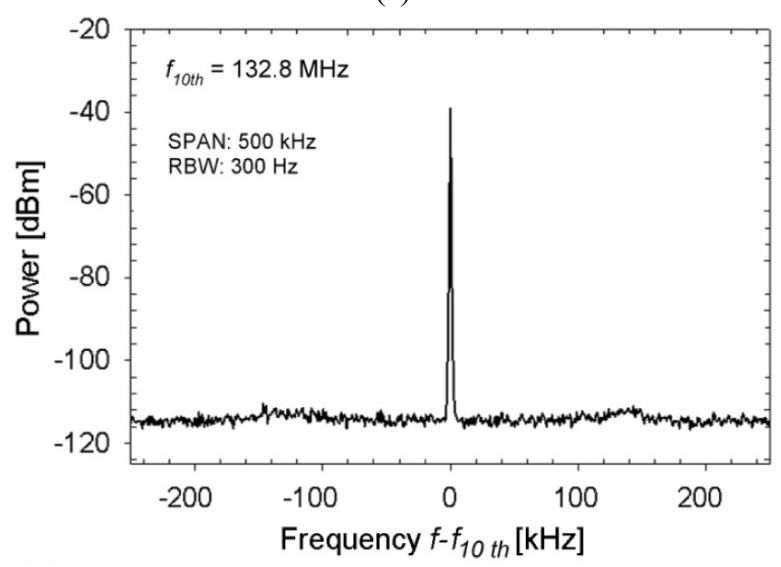

(b)

FIG. 10. RF spectra of the PMLFL with TBPF2 at the fundamental cavity frequency of $13.28 \mathrm{MHz}$ and (b) at the 10th harmonic frequency of $132.8 \mathrm{MHz}$.

$0.08 \%$ [46]. This also highlights the high stability of the PMLFL, considering that no additional measure was implemented to stabilize the PMLFL system besides utilizing a SESAM [49, 50].

Direct amplification of picosecond-pulses in a MOPA configuration:

As discussed above, a SESAM-based PMLFL could generate well-stabilized optical pulses in both femtosecond and picosecond regimes, which is absolutely suitable for use as a master oscillator to other laser systems. Here, we briefly discuss one application of the developed source to a large-core EDF-based MOPA system [37].

Figure 11 shows the MOPA system designed for generating wavelength-tunable picosecond pulses of $>10-\mathrm{kW}$ peak power. The system was seeded by the SESAM-based PMLFL with TBPF2 as described previously. It is noteworthy that the PC1 and PC2 were further adjusted in order to make the pulsewidth fixed at $\sim 8.5$ ps. Then, the output signal of the master-oscillator PMLFL was amplified up to $\sim 31 \mathrm{~mW}$ in terms of average power by a pre-amplifier based on a

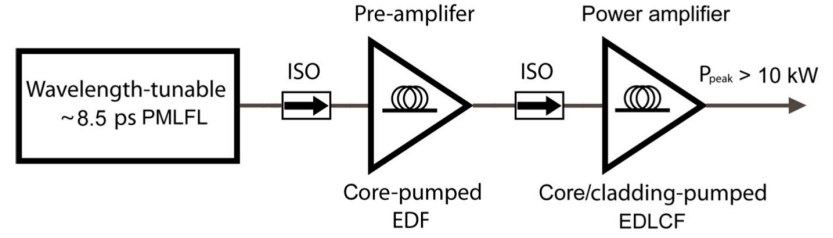

FIG. 11. Schematic of the MOPA system based on a picosecond-PMLFL (master oscillator) and EDF/EDLCF amplifiers. ISO: isolator.

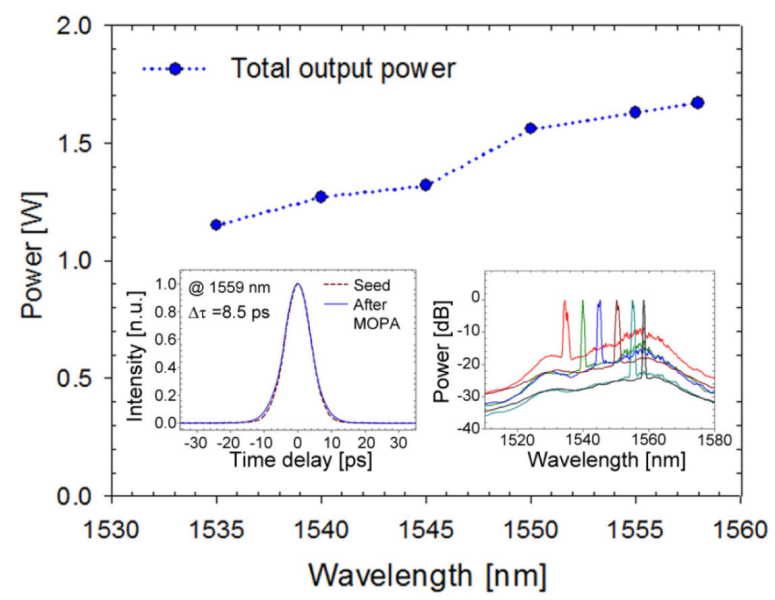

FIG. 12. Measured average output power for the whole tuning range of the MOPA system. Left inset: Autocorrelation traces of the seed and the output signal of the MOPA system tuned at $1559 \mathrm{~nm}$. Right inset: Normalized tuned output spectra (spectral resolution: $0.5 \mathrm{~nm}$ ).

core-pumped EDF (Fibercore) with a length of $1.3 \mathrm{~m}$. This pre-amplified signal was further amplified by a power amplifier comprising a $3.7-\mathrm{m} \mathrm{Yb}$-free, double-clad erbium-doped large-core fiber (EDLCF, CorActive). We utilized a hybrid pumping scheme to pump this double-clad EDLCF by using a multimode LD of $\sim 7.2 \mathrm{~W}$ at $976 \mathrm{~nm}$ and a single-mode Raman fiber laser of $\sim 950 \mathrm{~mW}$ at $1480 \mathrm{~nm}$. (See Ref. [37] for more details.)

Figure 12 shows the average output power measured over the entire tuning range, together with a typical autocorrelation trace of the output pulse at $1559 \mathrm{~nm}$ and its tuned spectra in the insets. The average output power was increased up to $1.67 \mathrm{~W}$ at $1559 \mathrm{~nm}$ while it was reduced to $1.15 \mathrm{~W}$ at $1535 \mathrm{~nm}$. Although the amplified spontaneous emission (ASE) in the power-amplifier stage was a limiting factor to the signal amplification for wavelengths shorter than $1550 \mathrm{~nm}$ [37], we emphasize that the stability and reliability of the master-oscillator PMLFL was well demonstrated over the entire tuning range of $1535 \mathrm{~nm}$ to 1559 $\mathrm{nm}$. In particular, the MOPA system could generate stable pulses with the maximum peak power of $\sim 12.6 \mathrm{~kW}$ and the pulse energy of $\sim 107 \mathrm{~nJ}$ when tuned at $1559 \mathrm{~nm}$. 
Wavelength-Tunable, Passively Mode-Locked Erbium-Doped Fiber … Luis A. Vazquez-Zuniga and Yoonchan Jeong 127

TABLE 3. Summary of the laser characteristics described in Section IV

\begin{tabular}{c|c|c|c|c|c|c|c}
\hline \hline $\begin{array}{c}\text { Laser } \\
\text { configuration }\end{array}$ & $\begin{array}{c}\text { Pulsewidth } \\
{[\mathrm{ps}]}\end{array}$ & $\begin{array}{c}\text { Wavelength } \\
{[\mathrm{nm}]}\end{array}$ & $\begin{array}{c}\text { Repetition rate } \\
[\mathrm{MHz})]\end{array}$ & $\begin{array}{c}\text { Output power } \\
{[\mathrm{mW}]}\end{array}$ & $\begin{array}{c}\text { Pulse energy } \\
{[\mathrm{pJ}]}\end{array}$ & $\begin{array}{c}\text { Amplitude } \\
\text { noise }[\Delta E / E]\end{array}$ & $\begin{array}{c}\text { Timing jitter } \\
{[\Delta T / T]}\end{array}$ \\
\hline $\begin{array}{c}\text { Fs-PMLFL } \\
\text { (w/o TBPF) }\end{array}$ & $0.248-0.385$ & 1556 & 18.30 & $\sim 1.9$ & $\sim 105$ & $0.091 \%$ & - \\
\hline $\begin{array}{c}\text { Ps-PMLFL } \\
\text { (w/TBPF1) }\end{array}$ & $2.76-11.30$ & $1533-1555$ & 12.87 & $\sim 0.4$ & $\sim 36$ & - & - \\
\hline $\begin{array}{c}\text { Ps-PMLFL } \\
\text { (w/TBPF2) }\end{array}$ & $8-10$ & $1535-1560$ & 13.28 & $\sim 1$ & $60-110$ & $0.47 \%$ & $0.08 \%$ \\
\hline Ps-MOPA & $\sim 8.5$ & $1535-1559$ & 13.28 & $\sim 1.6 \mathrm{~W}$ & $\sim 107 \mathrm{~nJ}$ & - & - \\
\hline
\end{tabular}

\section{CONCLUSION}

We have discussed SESAM-based PMLFLs, paying particular attention to an EDF-based system that was wavelength-tunable in the $1.5-\mu \mathrm{m}$ spectral range. The proposed simple and compact laser cavity enabled the PMLFL to operate either in a femtosecond regime or in a wavelength-tunable picosecond regime, both of which offered self-starting, stable CW ML pulses with no Q-switching instabilities. In a free-running cavity configuration without incorporating a TBPF, we could obtain stable 248-385 fs pulses, and the amplitude fluctuation was as low as $\sim 0.091 \%$ for 310 -fs pulses. In TBPF-inserted cavity configurations, the IL of the TBPF had a significant influence on the overall stability and tuning range of the PMLFL: With TBPF1 (IL $=\sim 5 \mathrm{~dB}$ ), we could obtain a stable tuning range of $1533 \mathrm{~nm}$ to 1555 $\mathrm{nm}$ for $\Delta \lambda_{F}=7.2 \mathrm{~nm}$. The advantage of this cavity configuration was that the pulsewidth was readily controllable from $\sim 2$ ps to $\sim 11$ ps via adjusting its filter bandwidth. With TBPF2 (IL $=\sim 1.5 \mathrm{~dB}$ ), we could continuously tune the PMLFL from $1535 \mathrm{~nm}$ to $1560 \mathrm{~nm}$ without having any interruption in the operating ML regime, where the amplitude fluctuation and timing jitter were given by $\Delta E / E=0.47 \%$ and $\Delta T / T=0.08 \%$, respectively. Furthermore, the excellent stability and reliability of the PMLFL eventually enabled it to be used as a master oscillator for a MOPA system which was based on a large-core $\mathrm{EDF}$, generating $\sim 8.5$-ps pulses of $>10-\mathrm{kW}$ peak power and $>100-n J$ pulse energy. Finally, Table 3 summarizes the laser characteristics of the PMLFLs developed and discussed in this paper. We think that these sources should be very effective for second-harmonic generation to a 770-780-nm spectral range or supercontinuum generation into a $2-\mu \mathrm{m}$ spectral range, for example, so that we expect they will have a lot of useful applications.

\section{ACKNOWLEDGMENT}

The work was supported in part by the Ministry of Knowledge Economy (Project No.10040429).

\section{REFERENCES}

1. J. M. Dudley, G. Genty, and S. Coen, "Supercontinuum generation in photonic crystal fiber," Rev. Mod. Phys. 78, 1135-1184 (2006).

2. R. R. Gattass and E. Mazur, "Femtosecond laser micromachining in transparent materials," Nature Photonics 2, 219-225 (2008).

3. M. E. Fermann and I. Hartl, "Ultrafast fiber laser technology," IEEE J. Select. Topics Quantum Electron. 15, 191-206 (2009).

4. J. Ye, H. Schnatz, and L. W. Hollberg, "Optical frequency combs: from frequency metrology to optical phase control," IEEE J. Select. Topics Quantum Electron. 9, 1041-1058 (2003).

5. G. Matthaus, B. Ortac, J. Limpert, S. Nolte, R. Hohmuth, M. Voitsch, W. Richter, B. Pradarutti, and A. Tünnermann, "Intracavity terahertz generation inside a high-energy ultrafast soliton fiber laser," Appl. Phys. Lett. 93, 261105-1 261105-3 (2008).

6. N. Nishizawa, Y. Chen, P. Hsiung, E. P. Ippen, and J. G. Fujimoto, "Real-time, ultrahigh-resolution, optical coherence tomography with an all-fiber, femtosecond fiber laser continuum at $1.5 \mu \mathrm{m}$," Opt. Lett. 29, 2846-2848 (2004).

7. J. Hansryd, P. A. Andrekson, M. Westlund, J. Li, and P. O. Hedekvist, "Fiber-based optical parametric amplifiers and their applications," IEEE J. Select. Topics Quantum Electron. 8, 506-520 (2002).

8. D. J. Richardson, J. Nilsson, and W. A. Clarkson, "High power fiber lasers: current status and future perspectives," J. Opt. Soc. Am. B 27, B63-B92 (2010).

9. L. E. Nelson, D. J. Jones, K. Tamura, H. A. Haus, and E. P. Ippen, "Ultrashort-pulse fiber ring lasers," Appl. Phys. B 65, 277-294 (1997).

10. K. Tamura, H. A. Haus, and E. P. Ippen, "Self-starting additive pulse mode-locked erbium fiber ring laser," Electron. Lett. 28, 2226-2228 (1992).

11. M. Hofer, M. E. Fermann, F. Haberl, M. H. Ober, and A. J. Schmidt, "Mode-locking with cross-phase and self-phase modulation," Opt. Lett. 16, 502-504 (1991).

12. H. A. Haus, E. P. Ippen, and K. Tamura, "Additive-pulse modelocking in fiber lasers," IEEE J. Quantum Electron. 30, 200-208 (1994).

13. M. E. Fermann, M. Hofer, F. Haberl, A. J. Schmidt, and L. Turi, "Additive-pulse-compression mode-locking of a neodymium fiber laser," Opt. Lett. 16, 244-246 (1991). 
14. D. J. Richardson, R. I. Laming, D. N. Payne, M. W. Phillips, and V. J. Matsas, "320 fs soliton generation with passively mode-locked erbium fiber laser," Electron. Lett. 27, 730-732 (1991).

15. U. Keller, D. A. B. Miller, G. D. Boyd, T. H. Chiu, J. F. Ferguson, and M. T. Asom, "Solid state low-loss intracavity saturable absorber for Nd-YLF lasers and antiresonant semiconductor Fabry-Perot saturable absorber," Opt. Lett. 17, 505-507 (1992).

16. U. Keller, "Recent developments in compact ultrafast lasers," Nature 424, 831-838 (2003).

17. U. Keller, K. J. Weingarten, F. X. Kärtner, D. Kopf, B. Braun, I. D. Jung, R. Fluck, C. Hönninger, N. Matuschek, and J. A. D. Au, "Semiconductor saturable absorber mirrors (SESAM's) for femtosecond to nanosecond pulse generation in solid-state lasers," IEEE J. Select. Topics Quantum Electron. 2, $435-453$ (1996).

18. B. C. Barnett, L. Rahman, M. N. Islam, Y. C. Chen, P. Bhattacharya, W. Riha, K. V. Reddy, A. T. Howe, K. A. Stair, H. Iwamura, S. R. Friberg, and T. Mukai, "High-power erbium-doped fiber laser mode locked by a semiconductor saturable absorber," Opt. Lett. 20, 471-473 (1995).

19. E. A. Desouza, C. E. Soccolich, W. Pleibel, R. H. Stolen, J. R. Simpson, and D. J. Digiovanni, "Saturable absorber modelocked polarization-maintaining erbium-doped fiber laser," Electron. Lett. 29, 447-449 (1993).

20. M. Guina, N. Xiang, A. Vainionp, O. G. Okhotnikov, T. Sajavaara, and J. Keinonen, "Self-starting stretched-pulse fiber laser mode locked and stabilized with slow and fast semiconductor saturable absorbers," Opt. Lett. 26, 1809-1811 (2001).

21. M. Guina, N. Xiang, and O. G. Okhotnikov, "Stretched-pulse fiber lasers based on semiconductor saturable absorbers," Appl. Phys. B 74, S193-S200 (2002).

22. O. G. Okhotnikov, L. Gomes, N. Xiang, T. Jouhti, and A. B. Grudinin, "Modelocked ytterbium fiber laser tunable in the 980-1070-nm spectral range," Opt. Lett. 28, 1522-1524 (2003).

23. M. Rusu, S. Karirinne, M. Guina, A. B. Grudinin, and O. G. Okhotnikov, "Femtosecond neodymium-doped fiber laser operating in the 894-909-nm spectral range," IEEE Photon. Technol. Lett. 16, 1029-1031 (2004).

24. R. Gumenyuk, I. Vartiainen, H. Tuovinen, and O. G. Okhotnikov, "Dissipative dispersion-managed soliton $2 \mu \mathrm{m}$ thulium/holmium fiber laser,” Opt. Lett. 36, 609-611 (2011).

25. M. Jiang, G. Sucha, M. E. Fermann, J. Jimenez, D. Harter, M. Dagenais, S. Fox, and Y. Hu, "Nonlinearly limited saturable-absorber mode locking of an erbium fiber laser," Opt. Lett. 24, 1074-1076 (1999).

26. A. Rutz, V. Liverini, R. Grange, M. Haiml, S. Schön, and U. Keller, "Parameter tunable GaInNAs saturable absorbers for mode locking of solid-state lasers," J. Cryst. Growth 301-302, 570-574 (2007).

27. O. G. Okhotnikov, T. Jouhti, J. Konttinen, S. Karirinne, and M. Pessa, "1.5 $\mu \mathrm{m}$ monolithic GaInNAs semiconductor saturable-absorber mode locking of an erbium fiber laser," Opt. Lett. 28, 364-366 (2003)

28. J. J. McFerran, L. Nenadovic, W. C. Swann, J. B. Schlager, and N. R. Newbury, "A passively mode-locked fiber laser at $1.54 \mu \mathrm{m}$ with a fundamental repetition fre- quency reaching $2 \mathrm{GHz}$," Opt. Express 15, 13155-13166 (2007).

29. F. X. Kärtner, I. D. Jung, and U. Keller, "Soliton modelocking with saturable absorbers," IEEE J. Select. Topics Quantum Electron. 2, 540-556 (1996).

30. L. Lefort, J. H. V. Price, D. J. Richardson, G. J. Spuler, R. Paschotta, U. Keller, A. R. Fry, and J. Weston, "Practical low-noise stretched-pulse $\mathrm{Yb}^{3+}$-doped fiber laser," Opt. Lett. 27, 291-293 (2002).

31. L. A. Gomes, L. Orsila, T. Jouhti, and O. G. Okhotnikov, "Picosecond SESAM-based ytterbium mode-locked fiber lasers," IEEE J. Select. Topics Quantum Electron. 10, 129-136 (2004).

32. S. Kivisto, J. Puustinen, M. Guina, O. G. Okhotnikov, and E. M. Dianov, "Tunable modelocked bismuth-doped soliton fibre laser," Electron. Lett. 44, 1456-1458 (2008).

33. R. C. Sharp, D. E. Spock, N. Pan, and J. Elliot, "190-fs passively mode-locked thulium fiber laser with a low threshold," Opt. Lett. 21, 881-883 (1996).

34. B. C. Collings, K. Bergman, S. T. Cundiff, S. Tsuda, J. N. Kutz, J. E. Cunningham, W. Y. Jan, M. Koch, and W. H. Knox, "Short cavity erbium/ytterbium fiber lasers mode-locked with a saturable Bragg reflector," IEEE J. Select. Topics Quantum Electron. 3, 1065-1075 (1997).

35. W. H. Loh, D. Atkinson, P. R. Morkel, M. Hopkinson, A. Rivers, A. J. Seeds, and D. N. Payne, "Passively mode-locked $\mathrm{Er}^{3+}$ fiber laser using a semiconductor nonlinear mirror," IEEE Photon. Technol. Lett. 5, 35-37 (1993).

36. L. A. Vazquez-Zuniga, "Ultrafast high power fiber lasers and their applications," Ph. D. Thesis, University of Southampton (2012).

37. L. A. Vazquez-Zuniga, H. Kim, and Y. Jeong, "Wavelengthtunable, picosecond fiber master-oscillator power amplifier source based on an erbium-doped large-core fiber," Opt. Commun. 294, 255-259 (2013).

38. S. Y. Set, H. Yaguchi, Y. Tanaka, and M. Jablonski, "Laser mode locking using a saturable absorber incorporating carbon nanotubes," J. Lightwave Technol. 22, 51-56 (2004).

39. S. Yamashita, Y. Inoue, S. Maruyama, Y. Murakami, H. Yaguchi, M. Jablonski, and S. Y. Set, "Saturable absorbers incorporating carbon nanotubes directly synthesized onto substrates and fibers and their application to mode-locked fiber lasers," Opt. Lett. 29, 1581-1583 (2004).

40. H. Zhang, D. Y. Tang, L. M. Zhao, and Q. L. Bao, "Large energy mode locking of an erbium-doped fiber laser with atomic layer graphene," Opt. Express 17, 17630-17635 (2009).

41. Z. P. Sun, T. Hasan, F. Torrisi, D. Popa, G. Privitera, F. Q. Wang, F. Bonaccorso, D. M. Basko, and A. C. Ferrari, "Graphene mode-locked ultrafast laser," ACS Nano 4, 803-810 (2010).

42. H. Zhang, D. Y. Tang, L. M. Zhao, Q. L. Bao, K. P. Loh, B. Lin, and S. C. Tjin, "Compact graphene mode-locked wavelength-tunable erbium-doped fiber lasers: from all anomalous dispersion to all normal dispersion," Laser Phys. Lett. 7, 591-596 (2010).

43. H. A. Haus, "Parameter ranges for CW passive modelocking," IEEE J. Quantum Electron. 12, 169-176 (1976).

44. BATOP Optoelectronics: http://www.batop.com/.

45. C. Hönninger, R. Paschotta, F. Morier-Genoud, M. Moser, and U. Keller, "Q-switching stability limits of continuous-wave 
Wavelength-Tunable, Passively Mode-Locked Erbium-Doped Fiber … Luis A. Vazquez-Zuniga and Yoonchan Jeong 129

passive mode locking,” J. Opt. Soc. Am. B 16, 46-56 (1999).

46. F. X. Kärtner, J. A. D. Au, and U. Keller, "Mode-locking with slow and fast saturable absorbers-What's the difference?" IEEE J. Select. Topics Quantum Electron. 4, 159-168 (1998).

47. S. M. J. Kelly, K. Smith, K. J. Blow, and N. J. Doran, "Average soliton dynamics of a high-gain erbium fiber laser," Opt. Lett. 16, 1337-1339 (1991).

48. D. V. D. Linde, "Characterization of the noise in continuously operating modelocked lasers,” Appl. Phys. B 39, 201-217 (1986).
49. I. Ozdur, M. Akbulut, N. Hoghooghi, D. Mandridis, S. Ozharar, F. Quinlan, and P. J. Delfyett, "A semiconductorbased 10-GHz optical comb source with sub 3-fs shot-noiselimited timing jitter and similar to $500-\mathrm{Hz}$ comb linewidth," IEEE Photon. Technol. Lett. 22, 431-433 (2010).

50. Y. S. Liu, J. G. Zhang, G. F. Chen, W. Zhao, and J. Bai, "Low-timing-jitter, stretched-pulse passively mode-locked fiber laser with tunable repetition rate and high operation stability,” J. Opt. 12, 0955204 (2010). 